\title{
Multisystem Inflammatory Syndrome Related to COVID-19 in Children
}

\author{
Itzhak Brook
}

Pediatric inflammatory multisystem syndrome temporally associated with severe acute respiratory syndrome coronavirus 2 (SARS-CoV-2) (PIMS-TS) is a rare but serious complication of coronavirus disease 2019 (COVID-19). Reports of PIMSTS started appearing around the world in the months after the pandemic began [1].

Two recent studies totaling 75 pediatric patients published in JAMA showed that PIMS-TS is novel and different from Kawasaki disease (KD) and toxic shock syndrome (TSS) [2, 3].

The study by Whittaker et al [2] characterized the clinical features of 58 children with PIMS-TS admitted to eight hospitals in UK from March 23 to May 16, 2020. The other study by Cheung et al [3] described 17 children and adolescents admitted to a single children's hospital in New York City with COVID-19, who developed PIMS-TS with features overlapping but identical to those of KD from April 18 to May 5, 2020.

In the study by Whittaker et al [2] patients' clinical features were compared with those of children diagnosed as having KD or KD shock syndrome [4]. Forty-five of 58 patients $(78 \%)$ had evidence of current or previous infection with the SARS-CoV-2 that causes COVID-19. Thirty-three (57\%) were females, and 40 (69\%) were black or Asian. Most children had been previously healthy, and only seven had an underlying condition such as asthma or epilepsy. All had signs of inflammation, including elevated levels of C-reactive protein, ferritin, and neutrophils.

All 58 patients had fever, 31 (53\%) had abdominal pain, $30(52 \%)$ had a rash, $26(45 \%)$ had conjunctivitis, $29(50 \%)$ developed shock and needed supportive drugs or fluids, 17 (29\%) had mucus membrane changes and cracked lips, 15 (26\%) had headache, nine (16\%) had enlarged lymph nodes, $13(22 \%)$ had kidney damage, nine (16\%) had swollen hands and feet, eight $(14 \%)$ had dilated coronary arteries or aneurysms, and six $(10 \%)$ had a sore throat. Four patients $(7 \%)$ developed abnormal heart rhythms. Twenty-five $(43 \%)$ required mechanical ventilation, and two (3\%) needed extracorporeal membrane oxygenation for severe heart dysfunction.

Thirteen individuals met the American Heart Association

Manuscript submitted June 23, 2020, accepted June 26, 2020

Published online July 1, 2020

Department of Pediatrics, Georgetown University School of Medicine, Washington, DC 20007, USA. Email: ib6@georgetown.edu

doi: https://doi.org/10.14740/ijcp389 criteria for KD [5], and 23 had fever and inflammation without characteristics of shock or KD. When compared with children with KD and TSS, PIMS-TS patients were older (median age: 9 vs. 2.7 years in KD and 3.8 years in TSS), and had elevated levels of C-reactive protein.

Whittaker et al [2] identified three unique features for presentations of PIMS-TS: 1) Persistent fever and high levels of inflammatory markers without features of KD, shock, or organ failure; 2) Characteristic signs and symptoms of KD; 3) Shock and clinical, echocardiographic, and lab evidence of heart damage.

They identified a pattern of cytokine expression that suggested an interferon signaling component, along with interleukin (IL)-6 and IL-10 production, similar to the one observed in KD and acute pulmonary COVID-19 infection. They concluded that the absence of elevated tumor necrosis factor (TNF)- $\alpha$ or IL-13 levels may differ from acute pulmonary COVID-19 infections [6].

All 17 patients in the study by Cheung et al [3] had a fever (median of 5 days), 14 had gastrointestinal symptoms, 12 had a rash, 11 had conjunctivitis, and nine had red, swollen lips. Three had low oxygen levels, and 13 went into shock. Fourteen had abnormal chest radiograph findings, and eight met the criteria for KD and five for incomplete KD [5].

All children had elevated levels of inflammatory markers, 16 had high serum IL-6 levels, 15 had high levels of Nterminal-pro-B-type natriuretic peptide (NT-proBNP), 14 had high levels of troponin T, 12 had reduced levels of lymphocyte white blood cells, and 11 had high levels of the immature white band cells.

Fifteen children required intensive care, and 10 needed support for low blood pressure. Nine patients had low oxygen levels, but none required mechanical ventilation. Three patients had abnormal heart rhythms, and one developed a medium-sized aneurysm.

Median patient age was 8 years (range: 1 - 16 years), nine were females, 12 were white, and all were previously healthy, with mild asthma in three, and their median hospital stay was 7.1 days.

The findings of these two studies can help clinicians characterize the clinical features of hospitalized, seriously ill children with PIMS-TS and provide insights into this apparently novel syndrome. The occurrence of abnormal cardiac findings suggests the need for long-term surveillance. Early recognition of PIMS-TS would enable pediatrician provide close monitoring and the optimal treatment. 


\section{Acknowledgments}

None to declare.

\section{Financial Disclosure}

None to declare.

\section{Conflict of Interest}

None to declare.

\section{Data Availability}

The author declares that data supporting the findings of this study are available within the article.

\section{References}

1. Zimmermann P, Curtis N. COVID-19 in children, pregnancy and neonates: a review of epidemiologic and clini- cal features. Pediatr Infect Dis J. 2020;39(6):469-477.

2. Whittaker E, Bamford A, Kenny J, Kaforou M, Jones CE, Shah P, Ramnarayan P, et al. Clinical Characteristics of 58 children with a pediatric inflammatory multisystem syndrome temporally associated with SARS-CoV-2. JAMA. 2020.

3. Cheung EW, Zachariah P, Gorelik M, Boneparth A, Kernie SG, Orange JS, Milner JD. Multisystem inflammatory syndrome related to COVID-19 in previously healthy children and adolescents in New York City. JAMA. 2020.

4. Li Y, Zheng Q, Zou L, Wu J, Guo L, Teng L, Zheng R, et al. Kawasaki disease shock syndrome: clinical characteristics and possible use of IL-6, IL-10 and IFN-gamma as biomarkers for early recognition. Pediatr Rheumatol Online J. 2019;17(1):1.

5. McCrindle BW, Rowley AH, Newburger JW, Burns JC, Bolger AF, Gewitz M, Baker AL, et al. Diagnosis, treatment, and long-term management of Kawasaki disease: a scientific statement for health professionals from the American Heart Association. Circulation. 2017;135(17):e927-e999.

6. Chen G, Wu D, Guo W, Cao Y, Huang D, Wang H, Wang $\mathrm{T}$, et al. Clinical and immunological features of severe and moderate coronavirus disease 2019. J Clin Invest. 2020;130(5):2620-2629. 